Document downloaded from:

http://hdl.handle.net/10251/121067

This paper must be cited as:

García-García, L.; Tomás Gironés, J.; Parra-Boronat, L.; Lloret, J. (2019). An m-health application for cerebral stroke detection and monitoring using cloud services. International Journal of Information Management. 45:319-327.

https://doi.org/10.1016/j.jinfomgt.2018.06.004

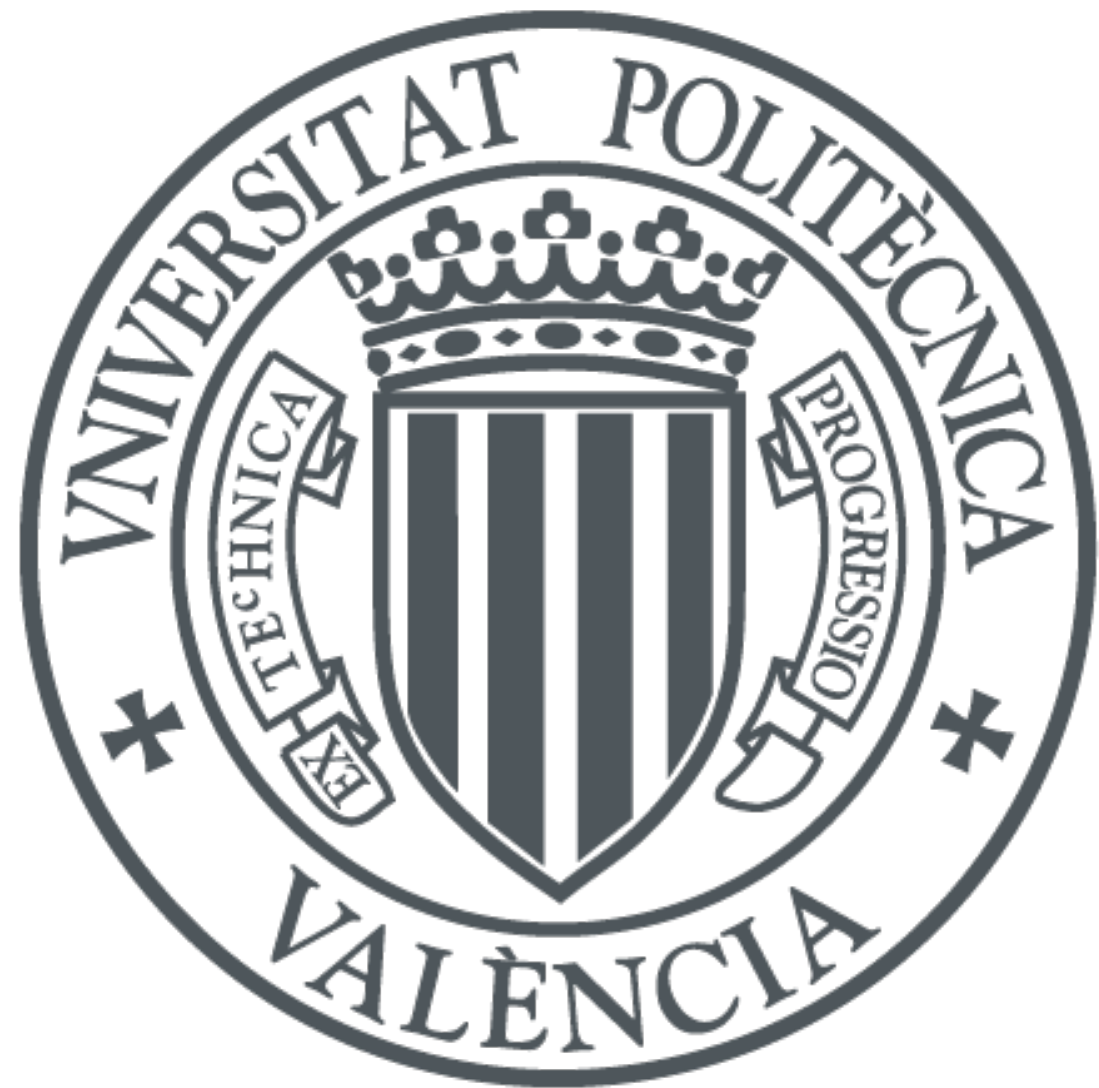

The final publication is available at

http://doi.org/10.1016/j.ijinfomgt.2018.06.004

Copyright Elsevier

Additional Information 


\title{
An M-health Application for Cerebral Stroke Detection and monitoring using Cloud Services
}

\author{
Laura García, Jesús Tomás, Lorena Parra, Jaime Lloret \\ Instituto de Investigación para la Gestión Integrada de zonas Costeras \\ Universitat Politècnica de València. C/ Paranimf, 1, 46730 Grau de Gandia, Valencia, Spain \\ laugarg2@teleco.upv.es, jtomas@upv.es, loparbo@doctor.upv.es, jlloret@dcom.upv.es
}

\begin{abstract}
Over 25 million people suffered from cerebral strokes in a span of 23 years. Many systems are being developed to monitor and improve the life of patients that suffer from different diseases. However, solutions for cerebral strokes are hard to find. Moreover, due to their widespread utilization, smartphones have presented themselves as the most appropriate devices for many e-health systems. In this paper, we propose a cerebral stroke detection solution that employs the cloud to store and analyze data in order to provide statistics to public institutions. Moreover, the prototype of the application is presented. The three most important symptoms of cerebral strokes were considered to develop the tasks that are conducted. Thus, the first task detects smiles, the second task employs voice recognition to determine if a sentence is repeated correctly and, the third task determines if the arms can be raised. Several tests were performed in order to verify the application. Results show its ability to determine whether users have the symptoms of cerebral stroke or not.
\end{abstract}

Keywords: Cerebral stroke, e-health, application, cloud, smile detection.

\section{INTRODUCTION}

Ischaemic strokes are one of the most common diseases in the world. From 1990 to 2013, 6.3 million people died from this type of stroke. Although the numbers are alarming, 25.7 million people were able to survive [1]. However, surviving a cerebral stroke does not guarantee a prompt recovery or a life exempt of the side effects caused by the lack of blood in the area of the brain affected during the stroke. Moreover, the fastest the stroke is detected, the least sequels the patient will have. The aftereffects of a cerebral stroke include a deterioration of cognitive and motor functions [2]. Memory loss, difficulties in speech, short attention spans, poor visuospatial skills or lack of orientations are some of the cognitive functions that can be impaired. Mobility in arms and legs is impaired as well resulting in an increased probability of falls [3]. Pain can also be present after the discharge from the hospital. Psychologically, patients may suffer from anxiety and depression. Numerous people do not know how to identify a cerebral stroke and many patients may spend more time than they should waiting for a family member or a person close to them to realize that they are having a stroke. Because of that reason, countless campaigns have been promoted by both hospitals and governments. However, as new platforms are available, the number of campaigns and solutions designed for mobile phones is increasing. 
Early disease diagnose of different diseases is increasingly being done utilizing Wireless Sensor Networks (WSN) [4]. They provide a great help to people who do not know the symptoms of a disease but suspect that they are having health related problems. Some countries experience overcrowded waiting rooms in hospitals [5]. Thus, these types of solutions may reduce the number of people that go to the emergency room as they may be advised by the application to go to a regular medical practice. Moreover, one of the areas that has had a greater growth in health-related technologies for health monitoring and prevention is Ambient Assisted Living (AAL) [6]. It provides varied features that allow to make the life of its user more comfortable such as turning on the lights when the user is approaching an area, turning off the television or other electronic devices and closing blinds or curtains at night. Furthermore, AAL in utilized as well to notify a trusted person or emergencies if the user is currently impaired. Falls, shouts or abnormal behavior can trigger the alarm system that contact the necessary parties [7]. Cloud solutions are often necessary in order to manage the vast volume of data generated by these applications [8]. Many providers have addressed this necessity and are now offering competitive services for IoT (Internet of Things) systems. The devices deployed at the house gather data through sensors and forward the information to a database. However, many health problems can also happen when being outside of home.

Smartphones provide a wide variety of sensors integrated in one device, allowing monitoring and measuring health at any place and any time. Multimedia sensors like the microphone and the camera integrated in smartphones allow to employ this device in a wide variety of e-health applications [9]. As a matter of fact, smartphone cameras were employed on a $67 \%$ of the e-health applications developed between 2010 and 2014, and 33\% of these applications employed the microphone. On the one hand, cameras can be employed for measuring heart rate, determine the emotions [10], detecting obstacles and diagnosing retinal and skin diseases. On the other hand, the microphone has been utilized to monitor nasal symptoms like sneezing, monitor sleep apnea, performing a spirometry or detecting stress. Non-multimedia sensors deployed in smartphones are employed for e-health applications as well. The accelerometer has also been widely utilized for e-health solutions. Body postures [11], falls [12] or activity recognition [13] are some of the activities that are usually monitored employing accelerometers. The conjunction of all these sensors are indicated for cerebral stroke detection as they allow developing the functionalities suitable for its symptoms.

In this paper, a mobile phone application for cerebral stroke detection is proposed. It allows to determine if the symptoms of cerebral strokes are being suffered by users. The application is able to contact a designated person by sending an SMS. Medical emergencies are also contacted in order to reduce the time the user is having a cerebral stroke without being treated. Moreover, demographic information on the user can be stored and analyzed in the cloud in order to provide statistics on the incidence of cerebral strokes. The contributions of this paper are:

- The proposal of a framework for our cerebral stroke detection system that employs cloud to store and analyze the data.

- An implementation of the prototype application which has allowed performing tests and evaluating the response of the user to the proposed application.

The rest of the paper is organized as follows. Section 2 depicts the related work. The proposal is described in section 3. Section 4 discusses the results. Finally, the conclusion and future work is presented in section 5 . 


\section{RELATED WORK}

In this section, we are going to present the state of the art on e-health smartphones applications, multimedia sensors and systems for e-health and cloud solutions for healthcare.

E-health solutions are increasingly being developed for mobile platforms as a great number of the population own mobile devices. A mobile application for obesity prevention was presented by Mohamed Alloghani et al. in [14]. It allowed to monitor food intake, location, the programmed diet and the advice provided by the doctor, among other features. It was aimed for children over 13 years old. The fitness status of the user was also able to be monitored evaluating data such as body weight, physical activities or burned calories. QR-codes were employed as well as a way of obtaining the nutrimental information of different food items. Andrea L. Hartzler et al. presented in [15] the use of NutriWalking, a mobile application that promoted exercise and healthy nutrition on patients with depression and type 2 Diabetes Metillus. The application provided a personalized plan for each user as well as goals and a progress tracker. Authors expected to further develop the app in order to feature a social network allowing the communication with other people suffering from the same health conditions. Saurav Gupta et al. designed in [16] a management system for personal health records called mSwasthya. Their system considered different aspects such as allergies, wellness, medicines, procedures or test details. Alerts were triggered by the parameters that surpassed their assigned threshold. They were notified through various systems including SMS, emails and push notifications. Moreover, Neha Nirwal et al. introduced in [17] Hopeful Hearts, a smartphone application for healthcare that provided suggestions on physical activity and diet. The application considered body temperature, heart rate, blood oxygen saturation, Body Mass Index (BMI), Basal Metabolic Rate (BMR) and working hours.

Multimedia sensors allow providing a wide variety of functionalities for health applications. Lorena Parra et al. performed in [9] a survey on multimedia sensors employed for e-health applications. A classification based on use is utilized. Authors discussed that the camera and the microphone are the most employed parts of a smartphone in health-related solutions. Sri Vijay Bharat Peddi et al. presented in [18] a cloud-based mobile application that employed the camera to detect the types of food on a plate and calculate the calories. There were three categories being single, multiple and mixed food objects. These allowed to differentiate different food items on the same plate. Moreover, they employed a cloud-broker mechanism that reduces the consumed time in a $77.21 \%$. A speech therapy game that employs Automatic Speech Recognition (ASR) was presented by Mario Ganzeboom et al. in [19]. The multimedia game was developed for mobile platforms, specifically for tablets from Apple. The Kaldi ASR toolkit was utilized in order to develop the application. Results show a word error rate of $32.1 \%$ for non-elderly people, $35.3 \%$ for elderly people and $31.2 \%$ for a combination of both non-elderly and elderly people. Furthermore, Haiyan Luo et al. presented in [20] a multimedia-based gait monitoring system that employs low-cost cameras to determine the behavior of the user. It was designed for clinics in order to be able to provide a faster diagnosis and prognosis. Moreover, the system extracted the human from the video so as to reduce the load on wireless networks. Results showed a $50 \%$ of video traffic reduction compared to the amount of traffic generated when the proposed system was not employed.

The computing requirements of multimedia solutions for healthcare have led to the employment of cloud services for e-health systems. Lo'ai A. Tawalbeh et al. performed in [21] a survey in mobile cloud computing and Big Data for health-related applications. Authors described the infrastructure of a mobile cloud computing infrastructure as well. They concluded that the necessity of computational and communication resources was the main reason for utilizing cloud in healthcare. Atif Almari proposed in [22] a multimedia framework based on cloud for e-health. His proposal employed scalable video coding so as to reduce the effort made by heterogeneous devices when processing videos. Simulation tests comparing PSNR (Peak Signal-to-Noise Ratio) 
and bitrate were performed in order to determine the performance of his solution. Results showed a better performance of his proposal over the anchor. Nimmy Jhon et al. described in [23] a cloudcomputing based healthcare system that was able to provide various types of services for health applications. The considered services were image processing, storage, analytics and reporting services. The image processing service was developed employing $\mathrm{C}++$ and Microsoft Windows Server was employed for the hosting service. Finally, Yin Zhang et al. proposed in [24] a CyberPhysical System (CPS) for healthcare that utilized cloud and Big Data in order to improve the performance of the healthcare system. Their system was composed of three functionalities. The first one was a data collection layer that acquired information on clinical data, expenses, research information and the activities and emotions of patients. The second one was a cloud platform for data storage and analysis. Finally, a user-interface and an API for developers was presented.

Although many solutions have been developed for different diseases, solutions for cerebral strokes are not common. As far as we know, no application has been developed to detect cerebral strokes using smartphones. There is a gap in the current available solutions for cerebral stroke detection that we are covering with this paper. In this paper, we present a cerebral stroke detection mobile application that employs the multimedia sensors on smartphones to identify the symptoms caused by cerebral strokes. Moreover, our proposal considers cloud services for data storing and analysis.

\section{PROPOSAL}

In this section, the proposed framework is going to be presented. Moreover, the prototype application is depicted as well.

\subsection{Proposed framework}

This subsection presents the proposed framework. The explanation of the functioning of the system is discussed as well.

People suffering from cerebral strokes present several symptoms that can help to identify their current health conditions. Although there are many of said symptoms, the most considered symptoms when teaching people how to identify cerebral strokes are three. The inability to smile is the most evident of the three of them. When being affected by a cerebral stroke, the mobility of half of the body is impaired. Facial muscles get affected making people unable to smile, often resulting in them making a grimace. The second symptom is related to the previous one. Raising both arms at the same level presents itself as a difficult task. The reduced mobility affects one of the arms, making it difficult to perform coordinated movements. Lastly, the third symptom is the lack of coherence in speech. The person is unable to answer simple questions or repeat a simple phrase. The combination of these three symptoms is a strong indicator of the presence of a cerebral stroke. When they are encountered, it is of great importance to contact medical emergencies as soon as possible, as time is an important factor in reducing the possible number of side effects or even avoiding death.

Our proposal is a cerebral stroke detecting mobile application. Our application considers the three aforementioned symptoms in order to make the user complete three tasks that are able to determine whether they are in the process of suffering from a cerebral stroke or not. Figure 1 presents the framework for our proposal. Our cerebral stroke detection application requires the user to perform the smile, raise your arms and speak tasks. If the application determines that the user is having a seizure, both medical emergencies and a family member or the selected contact number are contacted. This way, cerebral strokes can be correctly identified in a fast way whether users have some knowledge of this health problem or not. However, our proposal includes a functionality that allows to collect data from users in order to obtain statistics on several aspects of the people affected by cerebral strokes. 
Hospitals, research institutes, universities or the government can benefit from the knowledge that can be acquired from e-health applications as it can help in determining possible factors that affect citizens in suffering from different diseases. Cloud solutions present an efficient method to store and analyze the Big Data obtained from a great number of users. This way, statistics can be easily acquired and accessed not only by users but by medical staff, researchers and policy makers that need this information in order to perform their studies or create new policies. Google, Microsoft, Amazon or IBM are some of the service providers that offer cloud solutions [25]. Our proposal considers data on the sex of the user, their age, family precedents, and healthy habits like exercising or having a good diet. A hybrid cloud stores the information on each user identifying them by ID. When a cerebral stroke is detected, a log is created on the database containing the time of the event and the information of the user. This log is employed to obtain statistics on the demographics of the people suffering from cerebral strokes and the possible contributing factors. Thus, the information of each user is private. However, the statistics are public to the institutions without providing the identity of the users. When considering scalability for large scale patient care, it is important to determine the specific needs for the database and data analysis services that have to be requested to the cloud service providers. Population size, the number of hospitals or ambulance services are some of the aspects to consider. Both people suffering from a cerebral stroke and people who suspect that they are suffering it may use the application. However, it is unlikely for there to be a sudden acute increase of people having cerebral strokes. Thus, network requirements to provide service to the system should not present dramatic changes.

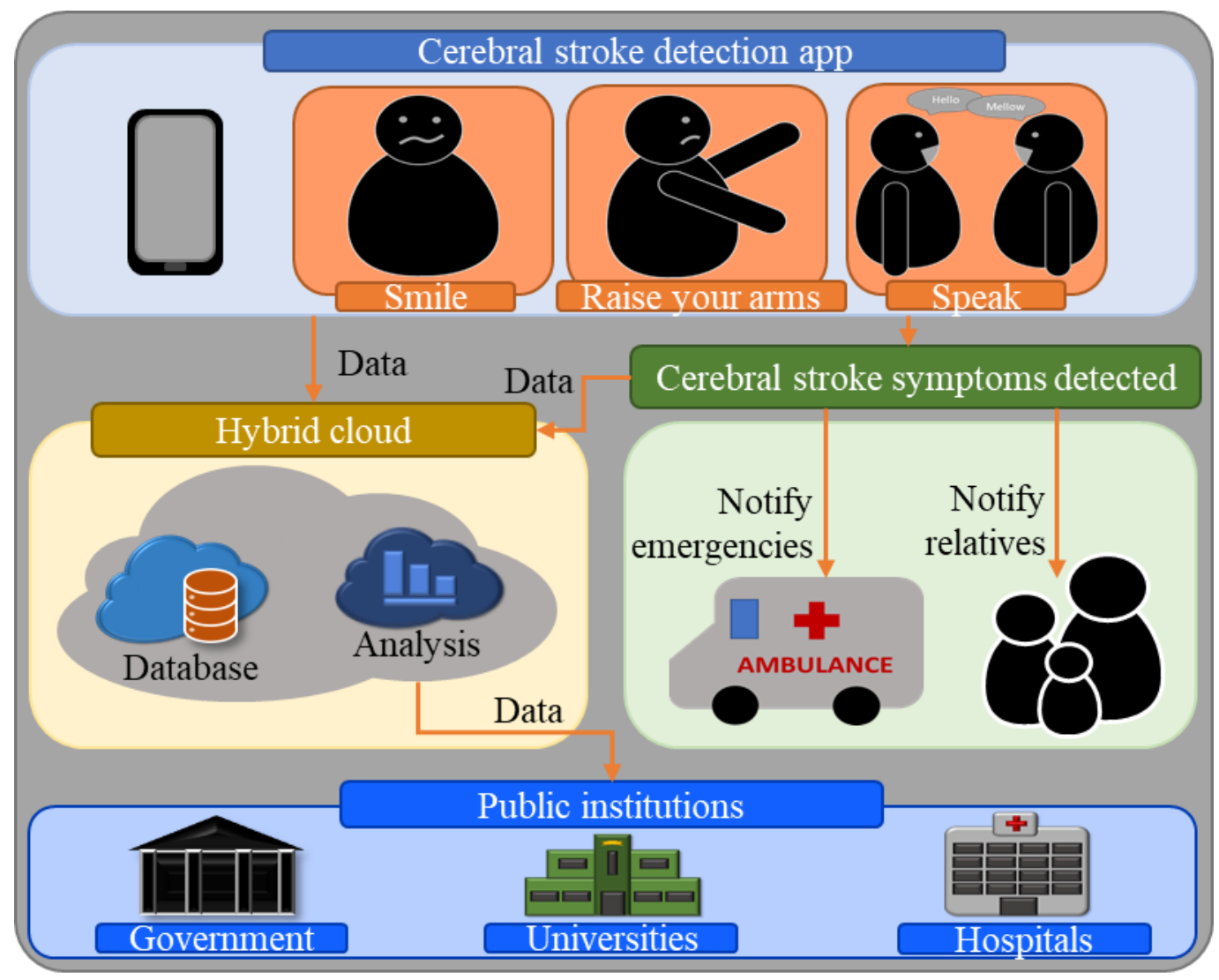

Figure 1. Proposed framework

The message exchange performed by the different agents of the framework is presented in Figure 2. In the setting up of the application the user registers the data on their age, sex, and other information on their activities and family precedents. The demographic data is then forwarded to the database. When the test is performed, if all tasks are completed, the favorable results are showed, and the data is forwarded to the database. If a cerebral stroke is detected, the unfavorable 
results are displayed, and a log is created in the database with the hour of the detection. Public institutions can request statistics. These statistics do not contain personal information on the patient in order to protect their identity. However, it is possible to obtain information on the sex, the age or the area were the most people suffering from cerebral strokes are. Moreover, it is also possible to obtain information on their diet and exercise habits. This information can be employed to develop cerebral stroke preventing policies.

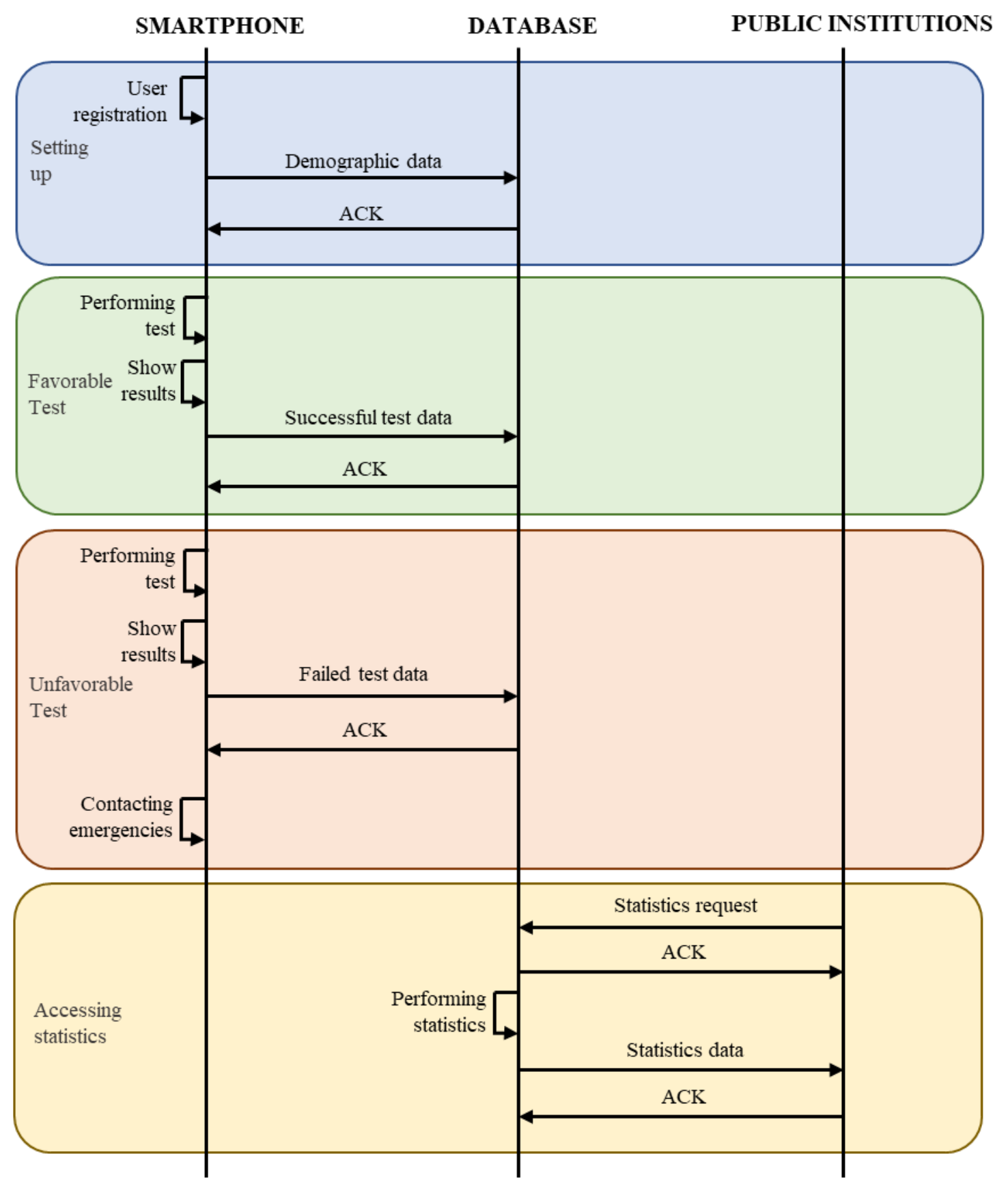

Figure 2. Message exchange between the agents of the framework

The algorithm that describes the performance of the application is depicted in Figure 3. When the application starts, the personal data of the user is requested. Then, the first task begins. The application asks the user to smile at the camera situated at the front of the phone. The application detects if the user is able to smile correctly. The results of the task are passed or not passed and are stored for further calculations. After obtaining the result of the first task $\left(T_{1}\right)$, the second task 
begins. The user is asked to say a simple phrase out loud. If the phrase is not spoken correctly, the task is not passed successfully. After the results of the second task $\left(T_{2}\right)$ are stored, the third task begins $\left(T_{3}\right)$. The user is asked to raise both arms. If the arms cannot be raised, the task is not successfully passed. With the information on the three tasks, a conditional evaluates if there are more than two failed tasks. If the result is true, a SMS is forwarded to the designated contact, the emergencies are contacted and, a summary of the results is displayed on the screen. If the result is false, the user is not considered to be suffering from a cerebral stroke and the app provides the results.

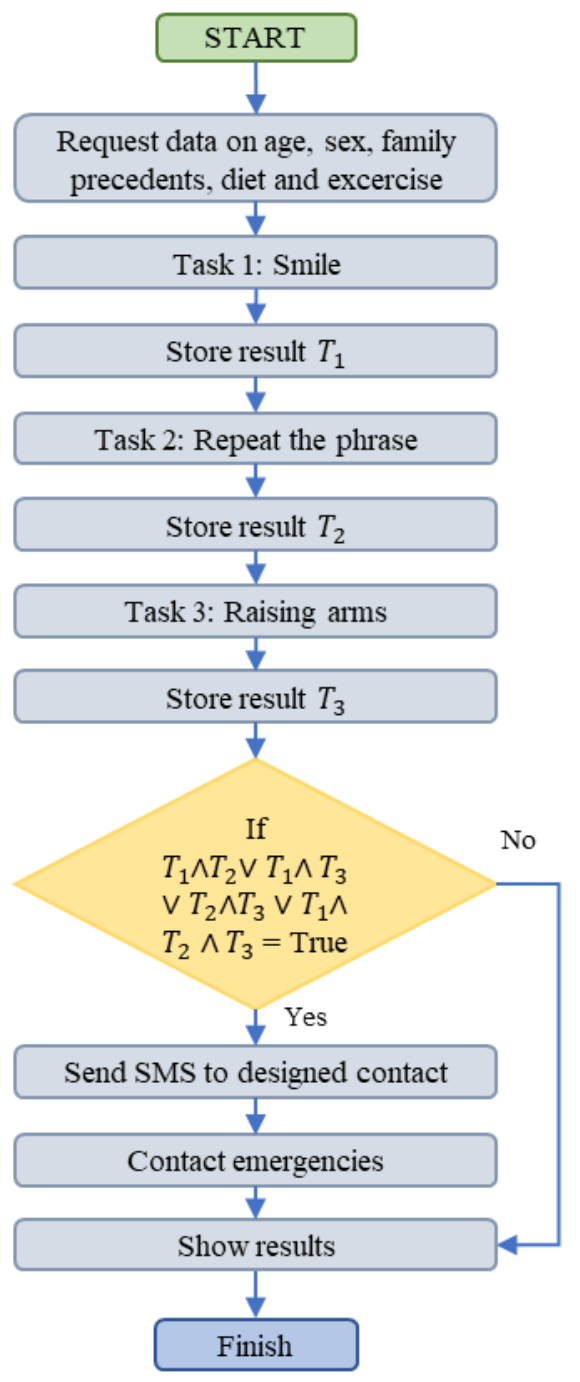

Figure 3. Algorithm of the application

\subsection{Prototype application}

The prototype of the application and its interface is presented in this subsection.

The application has been developed for Android employing the Android Studio development software. It has been debugged with the Android API 26 (v8.0 Oreo) SDK. Moreover, the employed tools have been the Android Mobile Vision API and the Android API for voice recognition and synthesis.

The user interface of the application is presented in Figure 4, whereas the result display is presented in Figure 5. Figure 4 a) presents the interface for the first task. The video image obtained from the camera is processed in order to detect smiles. When a smile is detected, the application 
automatically displays the second task as presented in Figure $4 \mathrm{~b}$ ). On the second task, the image of the speaker has to be pressed and a simple phrase has to be repeated. When the phrase is correctly spoken the application displays the third task as presented in Figure $4 \mathrm{c}$ ). On the third task the phone must be hold on one hand vertically and be raised until it ends up in a horizontal position following the natural arch described by the arms when performing the movement of raising your arms.

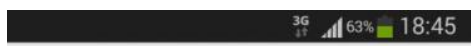

SMILE TO THE CAMERA

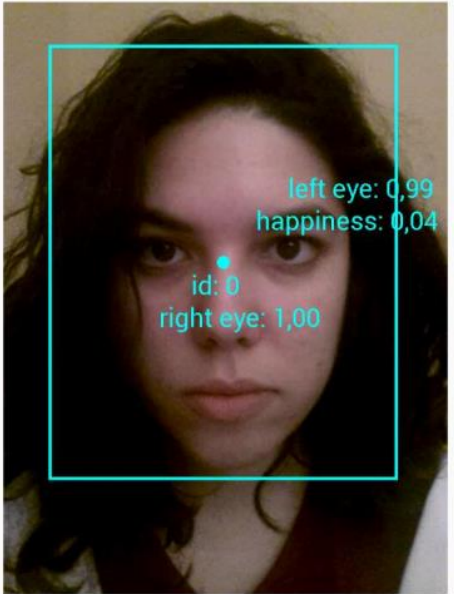

PASS

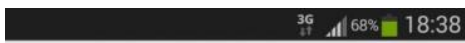

REPEAT AFTER ME

Tengo que comprar una docena de huevos

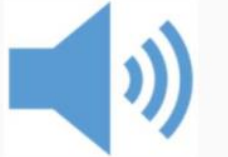

Press on the speaker and repeat.

PASS
RAISE YOUR ARMS

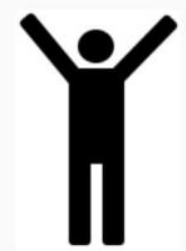

MIND THE POSITION OF THE PHONE

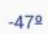

PASS

a)

b)

c)

Figure 4. User interface for the three tasks

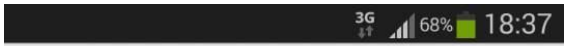

RESULTS

SMILE TO THE CAMERA

REPEAT AFTER ME

RAISE YOUR ARMS
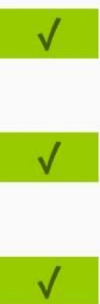

SMILE TO THE CAMERA

REPEAT AFTER ME

RAISE YOUR ARMS
RESULTS

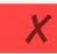

\section{$x$}

\section{$x$}

CONTANTING

EMERGENCIES

b)

Figure 5. Results display for both a positive and a negative result 
After performing the three tasks, the results are displayed on the screen. Figure 5 a) presents the results screen when all tasks are passed successfully. Figure $5 \mathrm{~b}$ ) presents the screen for the failed results. As it can be seen, the application notifies the user when the medical emergencies are being contacted. The results screen can also display one passed task and two failed ones and vice versa. When there are at least two failed tasks, the application contacts emergencies, displaying the red notification on the screen.

\section{RESULTS}

In this section, the system verification and results are presented. For evident reasons, people currently suffering from cerebral strokes were not sought after to perform the tests. Instead, tests were performed with a set of volunteer healthy people that were asked to perform the tests correctly or incorrectly.

A total of 90 tests were performed for both male and female volunteers. We recruited 5 different volunteers including three male volunteers and two female volunteers. The age of the youngest volunteer was 25 years old, while the age of the oldest volunteer was 45 years old.

First, we are going to present the results in terms of cerebral stroke detection. From the 90 performed tests, 16 tests were performed correctly, and the other 74 ones were performed incorrectly. The results offered by the application as cerebral stroke detection, correspond with the intentionality of the test. Table 1 presents the results of the success of the application for detecting if the tests were performed correctly interpreting it as "cerebral stroke $=$ false", or if the tests were performed incorrectly and then, it is considered as "cerebral stroke = true". There was no false positive or negative in the global test. Thus, we can affirm that this application can be used to detect if a patient may be having a cerebral stroke or not.

Table 1 Results of $\%$ of success of the developed application to detect cerebral stroke

\begin{tabular}{|c|c|c|c|}
\hline Test performed & cerebral stroke $=$ true & cerebral stroke $=$ false & \% of success \\
\hline Correctly & $0 / 0$ & $16 / 16$ & $100 \%$ \\
\hline Incorrectly & $74 / 74$ & $0 / 0$ & $100 \%$ \\
\hline
\end{tabular}

Thereupon, we are going to analyze individually the results of each one of the tasks requested by the application. The smile task is the first one. We are going to analyze the \% of achievement for this task. From the 90 tests, in 48 of the cases the volunteers were asked to perform the task correctly. They smiled to the camera as they usually smile. In the other 42 cases, the volunteers were asked to do not pass the test. They did not smile as usually, they just smiled with only one side of the face or did not smile at all. In Table 2, we show the $\%$ of success when detecting the smile. As it can be seen, in all the cases the application detected correctly if the volunteers were smiling or not. Thus, the $\%$ of success of the application for the correct tagging of the first task is $100 \%$. Moreover, we will analyze the time consumed by the volunteers to perform this task. The time consumed to perform the task 1 is shown in Figure 6. We can see that when the task is performed correctly (blue dot in position $=1$ ), smiling to the camera, the time consumed was lower than the time consumed when the task was not achieved (blue dot in position $=0$ ). The time consumed when the task is not achieved is $15000 \mathrm{~ms}$ in all the cases. The reason is because it is the maximum time that the application waits until it considers the task failed. When the task is performed correctly, time can have values lower than $15000 \mathrm{~ms}$. The mean value of consumed time when the task is achieved is $3205 \mathrm{~ms}$ with a standard deviation of $1510 \mathrm{~ms}$. The maximum and minimum time consumed for achieving the task 1 is $10037 \mathrm{~ms}$ and $1811 \mathrm{~ms}$. In Figure 7 we can see the maximum and minimum time consumed as a black car and the mean time consumed as a grey dot. When the test is passed, the used time is variable, the mean time is $3205 \mathrm{~ms}$, the 
median time $2649 \mathrm{~ms}$ and the standard deviation is 1510 . When the test is not passed the used time is always $15000 \mathrm{~ms}$, almost 5 times higher than the mean time if the test is passed.

Table 2 Results of $\%$ of success of the developed application in task 2

\begin{tabular}{|c|c|c|c|}
\hline Test performed & Task 1 = true & Task 1 = false & \% of success \\
\hline Correctly & $0 / 0$ & $48 / 48$ & $100 \%$ \\
\hline Incorrectly & $42 / 42$ & $0 / 0$ & $100 \%$ \\
\hline
\end{tabular}

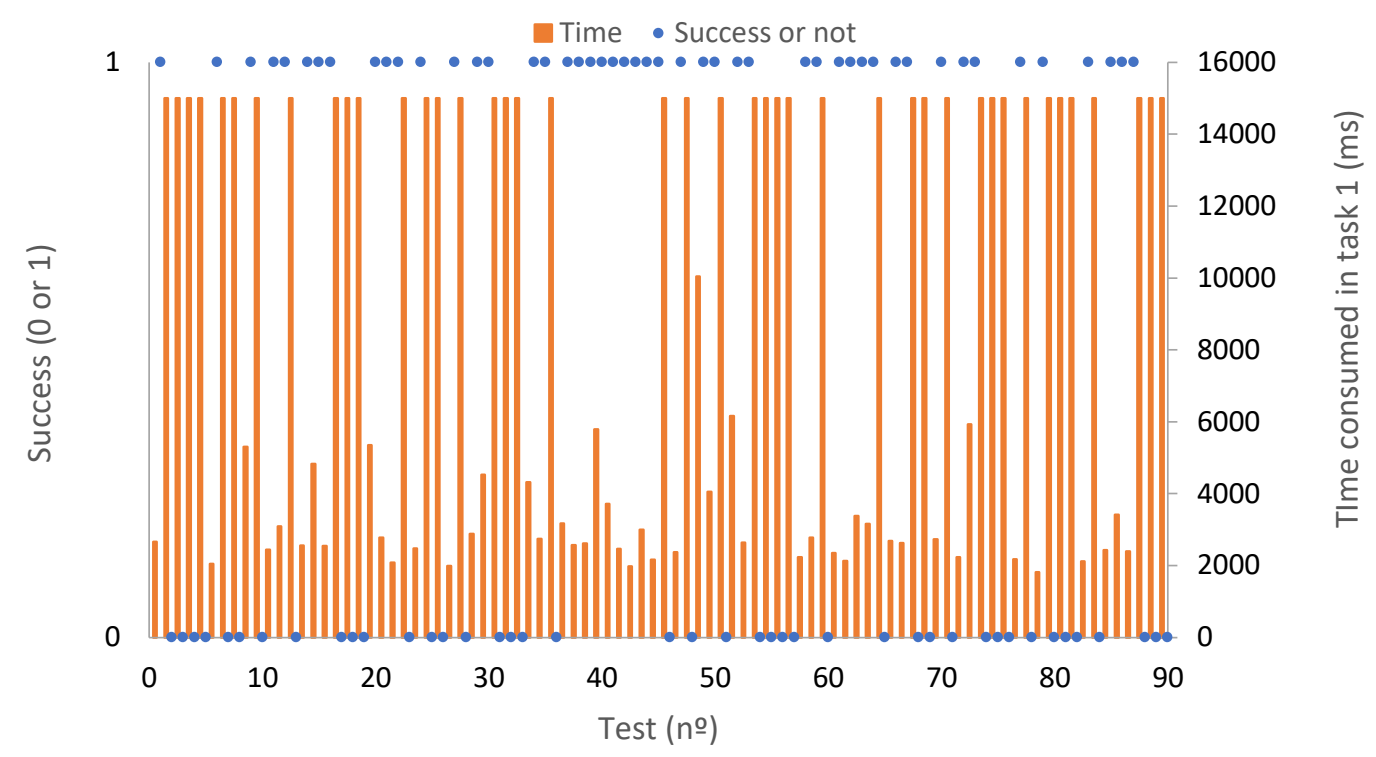

Figure 6. Time consumed to perform task 1

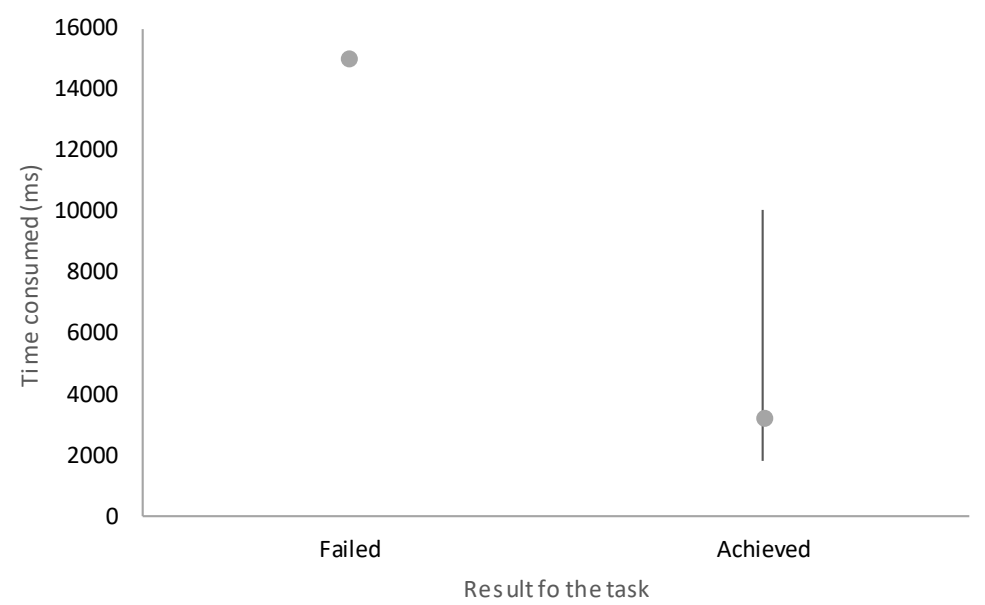

Figure 7. Time consumed in task 1 when fail and when achieve

Next, we are going to describe the results of the second task. In this task the volunteers where asked to repeat the sentence when they had to fulfill the task correctly, or to say anything else when they were asked to fail. From the 90 tests, in 47 of the cases we asked the volunteers to pass the test and, in the other 43 cases, we asked them to fail. In Table 3 we show the $\%$ of success when detecting the repeated sentence. Thus, the $\%$ of success of the application for the correct tagging of the second task is $100 \%$. In addition, we are going to analyze the time consumed by the volunteers to perform task 2 . In Figure 8 the time consumed in each test is shown, indicating with blue dots if the test was passed or not. The developed system allows the user to repeat the 
sentence if it was not clear. The accumulation of time consumed in performing all the aforementioned process is shown in Figure 8. In two cases, tests $n^{\circ} 2$ and test $n^{\circ} 6$, we asked the volunteer to just pass the test without saying anything. It was done to simulate the case where the patient cannot speak and the pass button is pressed. The maximum time consumed was $53040 \mathrm{~ms}$. And the average item was $19490 \mathrm{~ms}$. Now, in Figure 9 we present the data from the tests that have performed only one attempt of repeating the sentence. This data includes 40 failed tests and 44 successful tests. The mean time consumed per successful test in task 2 is $19967 \mathrm{~ms}$. The maximum and minimum time consumed were $36883 \mathrm{~ms}$ and $10314 \mathrm{~ms}$. For the tests that failed task 2 , the mean consumed time was $18465 \mathrm{~ms}$. The maximum and minimum time were $30568 \mathrm{~ms}$ and $18465 \mathrm{~ms}$. In this case there are no clear differences between the consumed time when the person achieves or fails the test.

Table 2 Results of \% of success of the developed application in task 2

\begin{tabular}{|c|c|c|c|}
\hline Test performed & Task 2 = true & Task 2 = false & \% of success \\
\hline Correctly & $0 / 0$ & $47 / 47$ & $100 \%$ \\
\hline Incorrectly & $43 / 43$ & $0 / 0$ & $100 \%$ \\
\hline
\end{tabular}

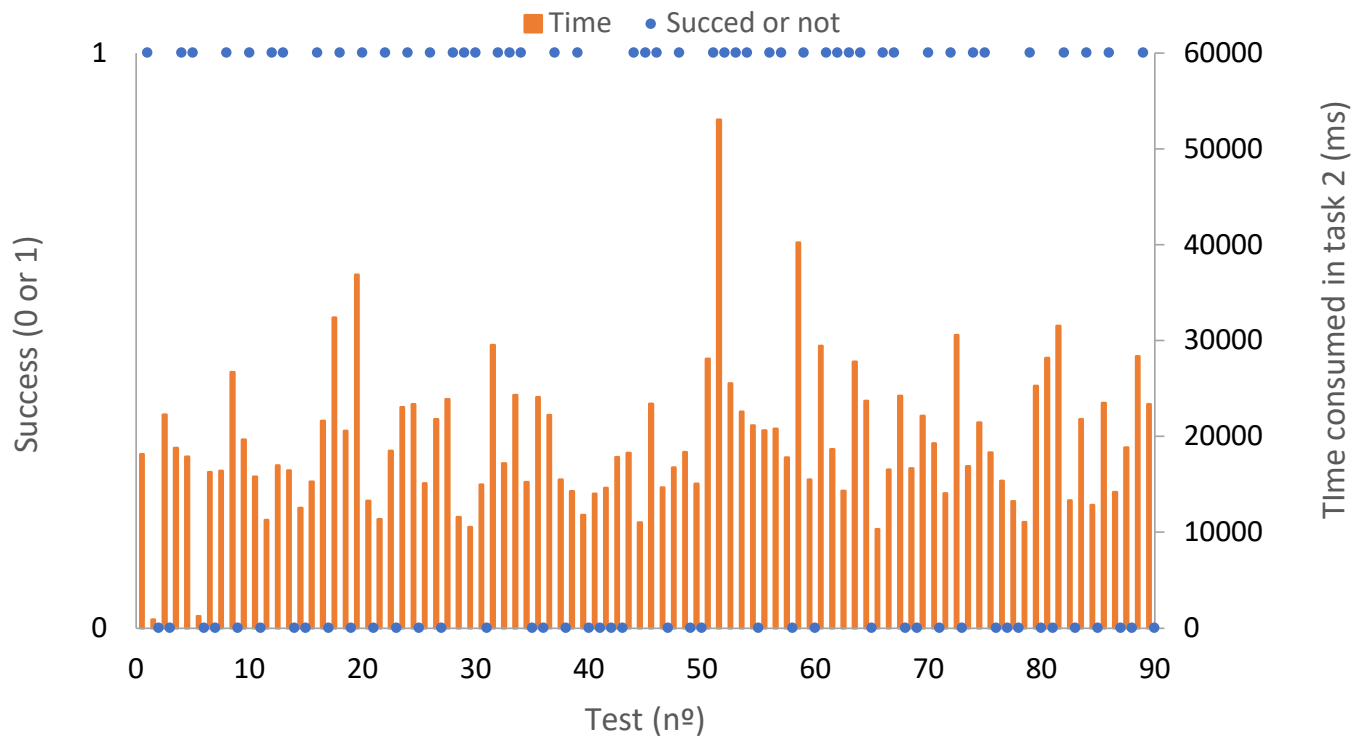

Figure 8. Time consumed to perform task 2

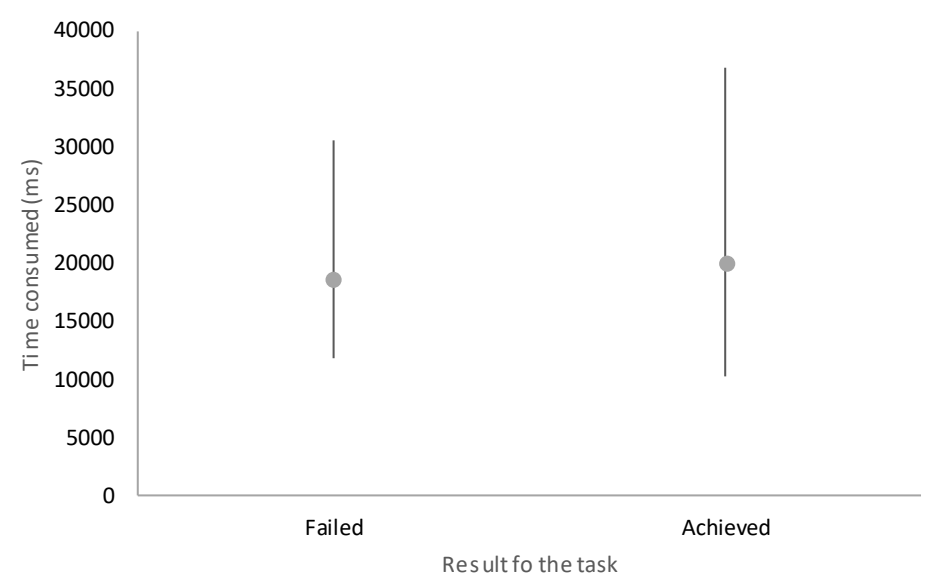

Figure 9. Time consumed in task 2 when fail and when achieve 
Now, the results of the third test are shown. In this test, the volunteers must rise their arms following the instructions. In 51 of the cases, the volunteers were asked to perform the movement correctly. In the other cases, the volunteers were asked to move their arms but not to complete the exercise successfully. In all the cases where the tests were performed correctly, the application tagged the test as successful. However, in one of the cases where the volunteer did not complete the exercise, the application tagged the test as successful. In the rest of the 38 cases, where the tests were performed incorrectly, the application tagged the test as failed. All this information is collected in Table 3. Figure 10 presents the time consumed in task 3 . The mean time consumed in test 3 was $116529 \mathrm{~ms}$ and the maximum was $207510 \mathrm{~ms}$. Then, we show the minimum time, maximum time, and mean time consumed in failed and successful tests, as shown in Figure 11. The maximum time consumed in a failed test was $207510 \mathrm{~ms}$ and for a successful test, it was $196950 \mathrm{~ms}$. The mean time for successful tests was $116444 \mathrm{~ms}$ and for the failed tests, it was $116641 \mathrm{~ms}$. The minimum consumed time for failed tests was $36869 \mathrm{~ms}$ and $23717 \mathrm{~ms}$ for successful tests. Again, in this case, the consumed time is almost the same for failed and for successful tests.

Table 3 Results of $\%$ of success of the developed application in task 3

\begin{tabular}{|c|c|c|c|}
\hline Test performed & Task 2 = true & Task 2 = false & \% of success \\
\hline Correctly & $0 / 0$ & $51 / 51$ & $100 \%$ \\
\hline Incorrectly & $38 / 39$ & $1 / 0$ & $97.4 \%$ \\
\hline
\end{tabular}

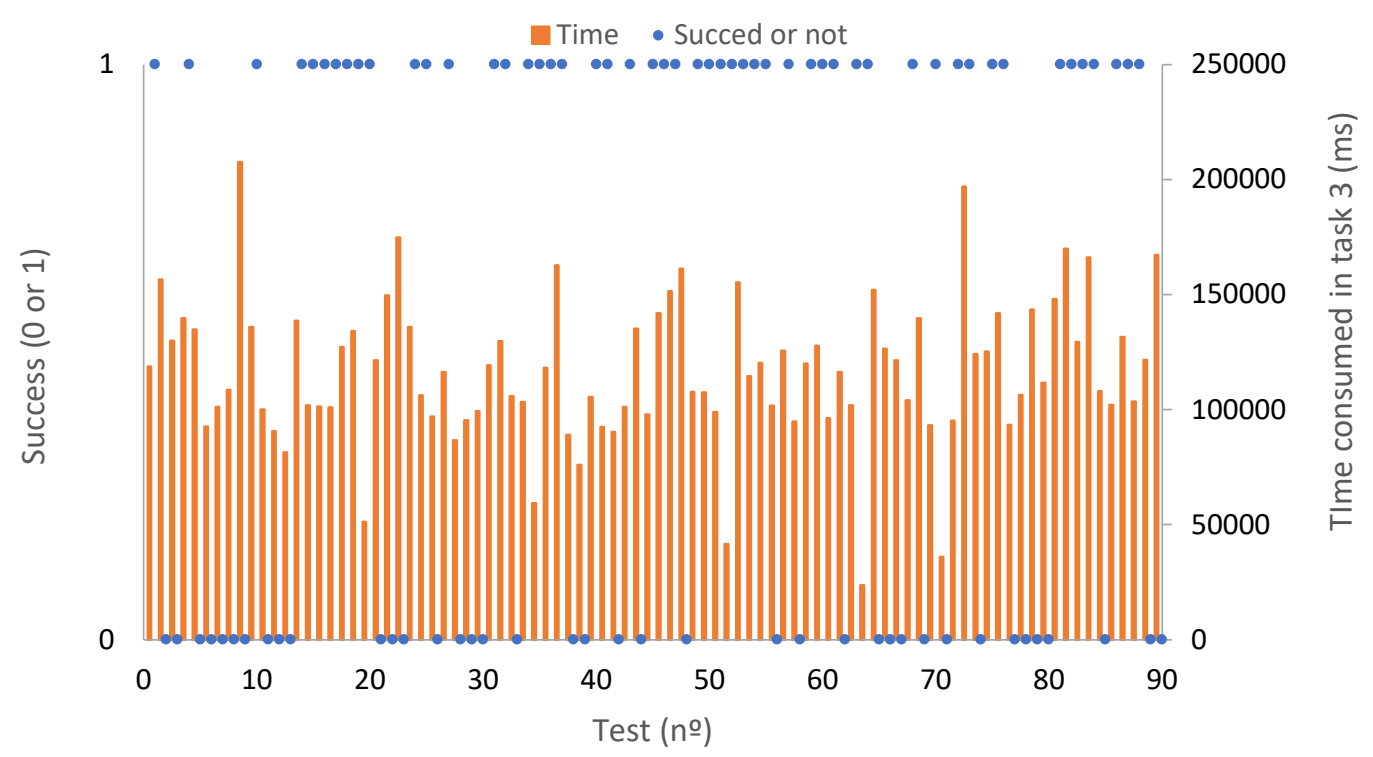

Figure 10. Time consumed to perform task 3

Finally, in Figure 12, we show the time consumed for all the tests. We can see that the task that requires more time to be performed is Task 3 . The maximum time consumed in the entire test was $239496 \mathrm{~ms}$ and the minimum time was $54669 \mathrm{~ms}$. The average time to perform the entire exercise is $144728 \mathrm{~ms}$. The fastest task is a successfully performed task 1 .

In addition, in order to ensure that the application is easy to use, other tests were done. In this case, the data was not gathered, and we only evaluate if people can follow the instructions and if the result was correct. Again, male and female volunteers used the application and followed the instructions. A total of 10 tests were done by different volunteers with ages between 50 and 60 years old. In this case, the volunteers were asked to perform the test correctly. In all the cases, the application succeeded in tagging the results of the test. 


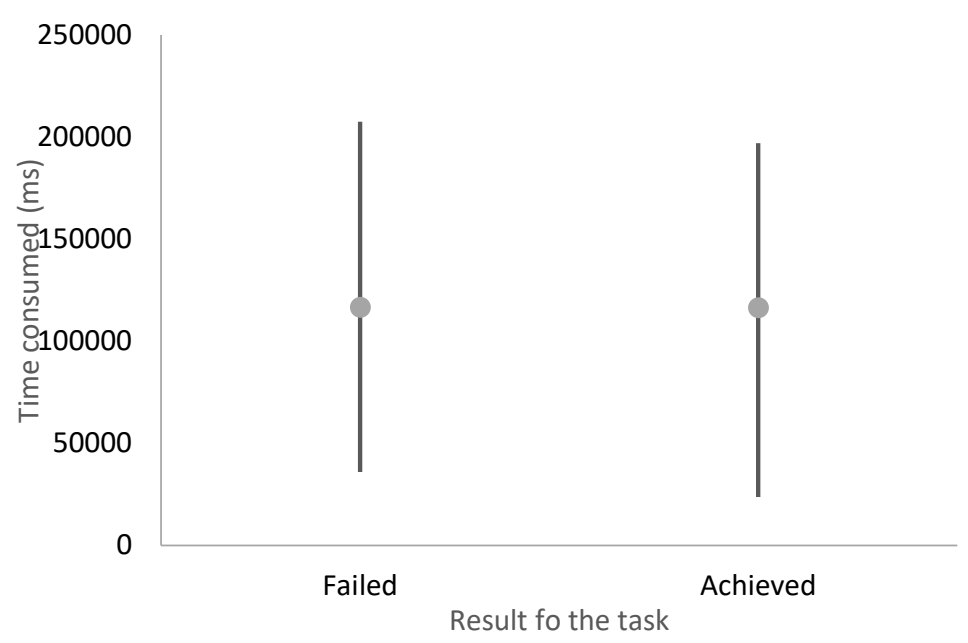

Figure 11. Time consumed in task 3 when fail and when achieve

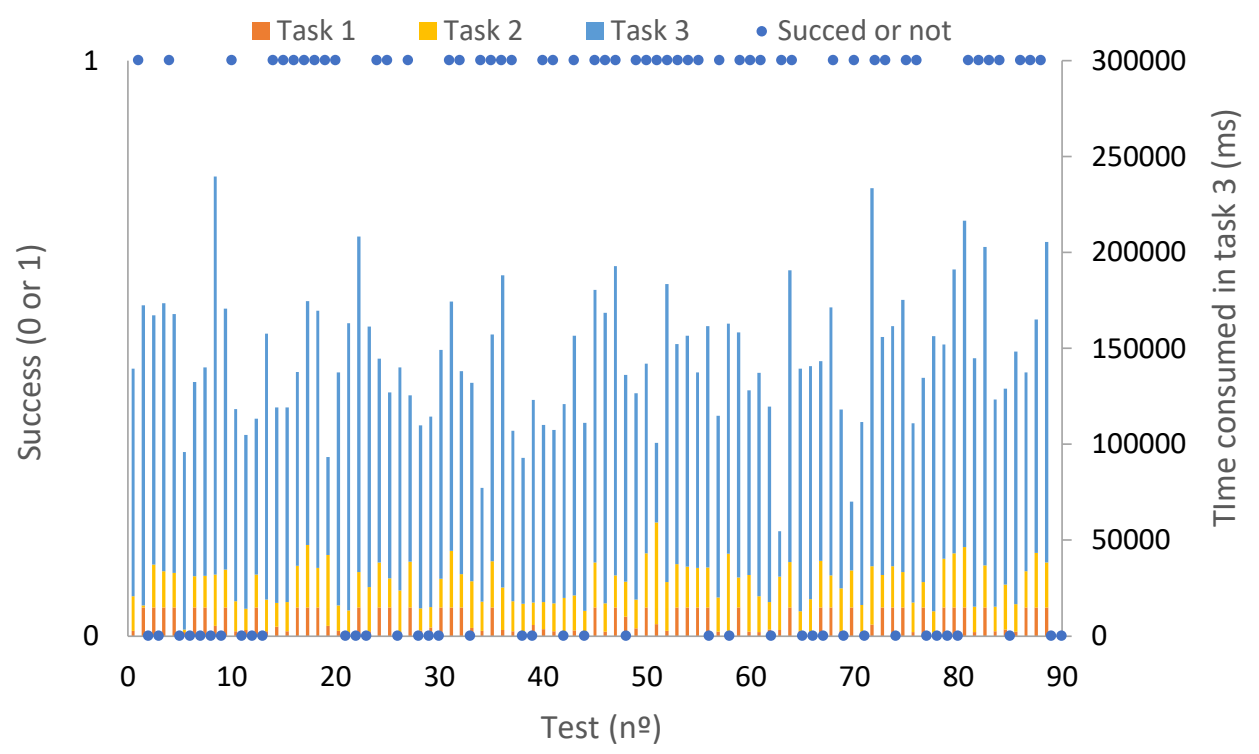

Figure 12. Time consumed to perform all the tasks required by the application

\section{CONCLUSION AND FUTURE WORK}

Cerebral strokes affect a great part of the population of the world. However, many people are not aware of its symptoms extending the time it takes to detect it and to get it treated. In this paper we propose a cerebral stroke detection mobile application. The user performs three tasks corresponding to the three most common symptoms of cerebral strokes. After being asked to smile, repeat a simple phrase and raise their arms successfully, the application provides the results and contacts family members and medical emergencies if necessary. Results provide the verification of our cerebral stroke detection application. Furthermore, the results on average time utilized for performing the test show $144728 \mathrm{~ms}$, being the smile detection task the fastest one and, the task of raising the arms, the one that takes the most time to be completed.

For future work we are planning to detect symptoms of other diseases in order to aid in the early detection of health conditions that need a prompt intervention. One of the conditions that we are planning to monitor is the asthma as in [26], fall detection [27], and obesity as it was done in [28]. 
That way, statistics on more conditions can be gathered and prevention campaigns can be more accurately developed. Especially we plan to apply the future solutions for kids as in [29], because they not be aware of the symptoms. Moreover, we also plan to include a privacy mechanism as in [30] to protect in a better way the data gathered from patients and consider new image processing mechanisms to add more functionalities to our solutions as in [31].

\section{ACKNOWLEDGMENT}

This work has been partially supported by the pre-doctoral student grant "Ayudas para contratos predoctorales de Formación del Profesorado Universitario FPU (Convocatoria 2014)" with reference: FPU14/02953 by the "Ministerio de Educación, Cultura y Deporte.

\section{AVAILABILITY OF DATA AND MATERIAL}

The datasets during and/or analyzed during the current study available from the corresponding author on reasonable request.

\section{References}

[1] J. F. Meschia and T. Brott, "Ischaemic stroke", European Journal of Neurology, 2017. DOI: 10.1111/ene.13409

[2] T. K. Tatemichi, D. W. Desmond, Y. Stern, M. Paik, M. Sano and E. Bagiella, "Cognitive impairment after stroke: frequency, patterns, and relationship to functional abilities", Journal of Neurology, Neurosurgery $\neg$ Psychiatry, Vol. 57, No. 2, pp. 202-207, 1994.

[3] P. Langhorne, D.J. Stott, L. Robertson, J. MacDonald, L. Jones, C. McAlpine, F. Dick, G.S. Taylor and G. Murray, "Medical Complications After Stroke: A Multicenter Study", Stroke, Vol. 31, No. 6, pp. 1223-1229, 2000.

[4] W. Chung, S. Lee and S. Toh, "WSN Based Mobile u-Healthcare System with ECG, Blood Pressure Measurement Function", Annual International Conference of the IEEE Engineering in Medicine and Biology Society, Vancouver, BC, Canada, 20-25 August 2008, pp. 1533-1536.

[5] O. M. Firdaus and T. M. A. A. Samadhi, "Conceptual model for reducing outpatient care waiting times in teaching hospital in Indonesia", IEEE International Conference on Management of Innovation and Technology, Singapore, Singapore, 23-25 September 2014, pp. 317-320.

[6] J. Lloret, A. Canovas, S. Sendra and L. Parra, "A smart communication architecture for ambient assisted living”, IEEE Communications Magazine, Vol. 53, No. 1, pp. 26-33.

[7] A. Rghioui, S. Sendra, J. Lloret and A. Oumnad, "Internet of Things for Measuring Human Activities in Ambient Assisted Living and e-Health", Network, Protocols and Algorithms, Vol. 8, No. 3, pp. 15-28, 2016.

[8] N. H. H. Cuong, V. K. Solanki, D. V. Thang and N. T. Thuy, "Resource Allocation for Heterogeneous Cloud Computing", Network, Protocols and Algorithms, Vol. 9, No. 1-2, pp. 7184, 2017.

[9] L. Parra, S. Sendra, J. M. Jiménez, J. Lloret, "Multimedia sensors embedded in smartphones for ambient assisted living and e-health" Multimedia Tools Applications, Vol. 75, pp. 13271132978, 2016. 
[10] D. Lakens, "Using a Smartphone to measure heart rate changes during relived happiness and anger", IEEE Transactions on Affective Computing, Vol. 5, No. 3, pp. 217-226, 2013.

[11] P. Patel, B. Bhatt and B. Patel, "Human Body Posture Recognition - a Survey", International Conference on Innovative Mechanisms for Industry Applications, Bangalore, India, 21-23 February 2017, pp. 473-477.

[12] Q. Li, J. A. Stankovic, M. A. Hanson, A. T. Barth, J. Lach and G. Zhou, "Accurate, Fast Fall Detection Using Gyroscopes and Accelerometer-Derived Posture Information", Sixth International Workshop on Wearable and Implantable Body Sensor Networks, Berkeley, CA, USA, 3-5 June 2009, pp. 138-143.

[13] W. Ugulino, D. Cardador, K. Vega, E. Valloso, R. Milidiú and H. Fuks, "Wearable computing: Accelerometers' Data Classification of Body Postures and Movements", Advances in Artificial Intelligence- SBIA 2012, Springer, Berlin, Heidelberg, pp. 52-61, 2012.

[14] M. Alloghani, A. Hussain, D. Al-Jumeily, P. Fergus, O. Abuelma'atti and H. Hamden, "A Mobile Health Monitoring Application for Obesity Management and Control Using the Internetof-Things", Sixth International Conference on Digital Information Processing and Communications, Beirut, Lebanon, 21-23 April 2016, pp. 19-24.

[15] A. L. Hartzler, A. Venkatakrishnan, S. Mohan, M. Silva, P. Lozano, J. D. Ralston, E. Ludman, D. Rosengerb, K. M. Newton, L. Nelson and P. Pirolli, “Acceptability of a Team-based Mobile Health (mHealth) Application for Lifestyle Self-management in Individuals with Chronic Illnesses", Orlando, FL, USA, 16-20 August 2016, pp. 3277-3281.

[16] S. Gupta, N. Kaur and D. K. Jain, "mSwasthya: a Mobile-enabled Personal Health Record Management System", International Conference on Computing, Comunication and Automation, Noida, India, 15-16 May 2015, pp. 374-379.

[17] N. Nirwal, N. Sardana and A. J. Bhatt, "Hopeful Hearts: A Mobile Health Care Application", Seventh International Conference on Contemporary Computing, Noida, India, 7-9 August 2014, pp. 351-356.

[18] S. V. B. Peddi, P. Kuhad, A. Yassine, P. Pouladzadeh, S. Shirmohammadi and A. A. N. Shirehjini, "An intelligent cloud-based data processing broker for mobile e-health multimedia applications", Future Generation Computer Systems, Vol. 66, pp. 71-86, 2017.

[19] M. Ganzeboom, E. Yilmaz, C. Cucchiarini and H. Strik, "On the development of an ASRbased Multimedia Game for Speech Therapy: Preliminary Results", ACM Worckshop on Multimedia for Personal Health and Health Care, Amsterdam, The Netherlands, 16 October 2016, pp. 3-8.

[20] H. Luo, S. Ci, D. Wu, N. Stergiou and K. Siu, "A Remote Markerless Human Gait Tracking for e-Healthcare Based on Content-aware Wireless Multimedia Communications", IEEE Wireless Communications, Vol. 17, No. 1, pp. 44-50, 2010.

[21] L. A. Tawalbeh, R. Mehmood, E. Benkhlifa and H. Song, "Mobile Cloud Computing Model and Big Data Analysis for Healthcare Applications", IEEE Access, Vol. 4, pp. 6171-6180, 2016.

[22] A. Almari, "Cloud-based E-health Multimedia Framework for Heterogeneous Network", IEEE International Conference on Multimedia and Expo Workshops, Melbourne, VIC, Australia, 9-13 July 2012, pp. 447-452. 
[23] N. John and S. Shenoy, "Health Cloud - Healthcare As A Service (HaaS)", International Conference on Advances in Computing, Communications and Informatics, New Delhi, India, 2427 September 2014, pp. 1963-1966.

[24] Y. Zhang, M. Qiu, C. Tsai, M. M. Hassan and A. Alamri, "Health-CPS: Healthcare CyberPhysical System Assisted by Cloud and Big Data", IEEE Systems Journal, Vol. 11, No. 1, pp. 8895, 2017.

[25] I. Mehmood, M. Sajjadd and S. W. Baik, "Mobile-Cloud Assisted Video Summarization Framework for Efficient Management of Remote Sensing Data Generated by Wireless Capsule Sensors", Sensors, Vol. 14, pp. 17112-17145, 2014.

[26] J. Lloret, L. Parra, M. Taha, J. Tomás, “An architecture and protocol for smart continuous eHealth monitoring using 5G”, Computer Networks, Vol. 129, 3pp. 40-351, 2017.

[27] P. Tsinganos, and A. Skodras, "On the comparison of wearable sensor data fusion to a single sensor machine learning technique in fall detection", Sensors, Vol. 18, No. 2, 592, 2018.

[28] M. S. Mohammed, S. Sendra, J. Lloret, I. Bosch, "Systems and WBANs for Controlling Obesity", Journal of healthcare engineering, Vol. 2018, pp. 1-21, 2018.

[29] S. Sendra, L. Parra, J. Lloret, J. Tomás, "Smart system for children's chronic illness monitoring”, Information Fusion, Vol. 40, pp. 76-86, 2018.

[30] H. S. Gardiyawasam Pussewalage and V. A. Oleshchuk, "Privacy preserving mechanisms for enforcing security and privacy requirements in E-health solutions", International Journal of Information Management, Vol. 36, No. 6, Part B, pp. 1161-1173, 2016.

[31] A. Karambakhsh, A. Kamel, B. Sheng, P. Li, P. Yang and D. D. Feng, "International Journal of Information Management, 2018. DOI: https://doi.org/10.1016/j.ijinfomgt.2018.03.004 